\title{
Fréquences d'application d'engrais minéraux: Impact sur les variables de croissance et de développement de la tomate hivernale (Lycopersicon esculentum Mill.) sous serre au centre du Burkina Faso.
}

\author{
Ali GARANE ${ }^{1 *}$, Koussao SOME ${ }^{1}$, Jeanne NiKIEMA $^{1}$, Koala OUANGO ${ }^{1}$ Mamoudou \\ TRAORE ${ }^{2}$ et Mahamadou SAWADOGO ${ }^{3}$ \\ ${ }^{1 *}$ Institut de l'Environnement et de Recherches Agricoles (INERA), Centre de recherche Environnementale, Agricole et de \\ formation de Kamboinsé (CREAF-Kamboinsé), Département Production Végétale, 01 BP 470 Onagadongou 01, Burkina \\ Faso, \\ Institut de l'Environnement et de Recherches Agricoles (INERA/CREAF-Kamboinsè), Centre de recherche \\ Environnementale, Agricole et de formation de Kamboinsé, Département Gestion des Ressources Naturelles/Système de \\ Production, 01 BP 470 Ouagadougou 01, Burkina Faso, \\ ${ }^{3}$ Université de Onaga I Pr Joseph Ki-Zerbo, Unité de Formation et de Recherche en Science de la Vie et de la Terre \\ (UO/UFR-SVT), Laboratoire de Génétique et Biotechnologie Végétale, 03 BP 7021 Onagadongou 03, Burkina Faso, \\ * Auteur correspondance: ali garane@yahoo.fr
}

Mots clés: Tomate, hivernage, fraction d'engrais, croissance, développement, productivité, Burkina Faso. Key words: Tomato, wintering, fertilizer fraction, growth, development, productivity, Burkina Faso

Publication date 30/09/2019, http://www.m.elewa.org/JAPS

\section{RESUME}

L'objectif de l'étude a été d'évaluer l'effet des fréquences d'apport des engrais minéraux sur les variables de croissance, de développement, de précocité et de productivité des variétés de tomate en culture sous abri en saison pluvieuse. Trois doses, de NPK et d'urée ont été apportées sur les plants de variétés «Padama», «Thorgal» et «Tomy» de tomate dans un essai sous abri de mai à août 2014 en régime pluvial. Le dispositif expérimental était un split-plot avec 4 répétitions, ayant pour facteur principal les trois fréquences de fertilisation et le facteur secondaire la variété. Les observations et mesures ont porté sur la hauteur et le diamètre de la tige principale des plants, les dates de floraison et de nouaison, la hauteur d'insertion du 1er bouquet floral, les nombres de fleurs et de grappes à fruit par plant. Nos résultats ont montré que les plants issus d'un apport de NPK et d'urée toutes les trois semaines (F2) ont cru plus rapidement que ceux qui étaient sous F1 (2 semaines) et F3 (apport unique). De même, cette fréquence d'apport a induit une hauteur d'insertion plus élevé du 1er bouquet floral chez tous les cultivars. Toutefois il a été constaté une similarité des diamètres de la tige principale sous les trois fréquences d'apport pour chaque variété. L'apport unique (F3) et toutes les deux semaines (F1) d'engrais NPK et d'Urée, ont augmenté la préciosité chez toutes les variétés par rapport à l'apport toutes les trois semaines (F2). Aucunes différences significatives entre les trois traitements pour le nombre de bouquets floraux et de grappes à fruits n'ont été observées. 
Mineral fertilizers application frequencies: impact on the growth and development parameters of winter tomato under greenhouse in central Burkina Faso

\begin{abstract}
The objective of the study was to evaluate the effect of mineral fertilizer input frequencies on growth, development, earliness and productivity variables of tomato varieties under rainy season cover crops. Three doses of NPK and urea were applied to tomato "Padama", "Thorgal" and "Tomy" seedlings in a shelter trial from May to August 2014 under rainfed conditions. The experimental design was a split-plot with 4 repetitions, with the main factor being the three frequencies of fertilization and the secondary factor the variety. Observations and measurements related to the height and diameter of the main stem of the plants, the dates of flowering and fruit set, the insertion height of the 1st floral bouquet, the numbers of flowers and fruit clusters per plant. Our results showed that plants fed with NPK and urea every three weeks (F2) grew faster than those under F1 (two weeks) and F3 (one application). Likewise, this feeding frequency induced a higher insertion height of the 1st floral bouquet in all cultivars. However, a similarity of the diameter of the main stem was found under the three delivery frequencies for each variety. The single (F3) and biweekly (F1) intake of NPK and Urea fertilizers increased the preciosity in all varieties compared to the intake every three weeks (F2). No significant differences between the three treatments for the number of flower bouquets and fruit clusters were observed.
\end{abstract}

\section{INTRODUCTION}

Au Burkina Faso, la pauvreté originelle des sols en éléments nutritifs justifie l'efficacité des engrais minéraux sur les rendements des cultures (Bado, 1994, Kabrah et al., 1996; Sedego et al., 1997). En effet, l'amélioration de la fertilité du sol par des apports d'éléments nutritifs sous formes minérales ou organiques permet d'augmenter l'efficience de l'eau et les rendements des cultures (Sedego et al., 1997). Cependant, l'utilisation des engrais minéraux sur les cultures vivrières en milieu rural, reste insignifiante compte tenu du faible pouvoir d'achat du paysan (Nyembo et al, 2013). Néanmoins, un effort est fait par les producteurs Burkinabè dans l'apport des engrais minéraux aux cultures maraîchères eu égard à la contribution financière de ces cultures dans l'amélioration de leurs revenus. En effet, la tomate au Burkina Faso occupe le premier rang sur le plan de la quantité produite (INERA, 2010) et sur le plan des revenus monétaires (MASA, 2012). La culture est essentiellement pratiquée en saison sèche en raison de l'absence ou de l'inaccessibilité de variétés adaptées à la saison pluvieuse. La quantité d'engrais apportée à cette culture de saison sèche est de $450 \mathrm{~kg} /$ ha de NPK et de $200 \mathrm{~kg} / \mathrm{ha}$ d'urée. Cette quantité est apportée en une seule application pour le NPK et en deux applications pour l'urée. La fertilisation minérale de cette production d'hivernage au niveau des producteurs est faite de la même manière que la production de saison sèche. Pourtant, si en saison sèche les apports d'eau sont contrôlés, limitant les pertes des éléments minéraux par lixiviation; ce n'est pas le cas en saison pluvieuse. En effet, les précipitations plus fortes et non contrôlées comme l'irrigation en saison sèche provoque une nitrification accélérée de l'azote et les pertes par lixiviation sont augmentées. Selon Pieri (1982), la quantité d'azote perdue en saison pluvieuse en sol sableux oscille entre 10 et $50 \mathrm{~kg} / \mathrm{ha}$. Cette lixiviation est d'autant plus accentuée que la hauteur d'eau à la surface du sol est élevée (Ganry, 1990). Dans le cas d'un sol ferrugineux tropical lessivé, $1 \mathrm{~mm}$ de pluie provoque un lessivage des nitrates vers les couches inférieures du sol qui peuvent descendre jusqu'à 70 mm (Ganry, 1990). A ces fortes lixiviations, 
s'ajoutent les pertes par ruissellement. Gigou et Chabalier (1987) ont observé des pertes d'azote par érosion hydrique d'environ $11 \mathrm{~kg} /$ ha sous culture de maïs. L'ion nitrate en est le principal élément perdu. Ces ions entraînent avec eux le calcium et le magnésium (Gigou et Chabalier 1987). En outre, même en saison sèche, la disponibilité des éléments apportés en début de végétation ne coïncide pas toujours avec les moments de forts besoins de la tomate. En effet, l'azote de par sa très forte mobilité dans le sol, son efficacité fertilisante et ses pertes sont associées à la composition des engrais et à leur période d'épandage (Nyembo et al., 2013). En général, l'ammoniac qui se trouve dans l'urée est fixé sous forme de $\mathrm{NH}_{4}^{+}$sur les fractions argileuses et sur la Matière Organique $(\mathrm{MO}) \mathrm{du}$ sol (FAO, 2003). Il est de même pour le phosphore et le potassium. Mais les sols du Burkina Faso étant très pauvres en matière organique (Bunasols, 1985), irrigation et/ ou les

\section{MATERIEL ET METHODES}

3.1 Brève description du milieu biogéographique : Selon Guinko (1984), le climat de Kamboinsé est de type Nord Soudanien car se trouvant entre les isohyètes 600 et $900 \mathrm{~mm}$. Il est caractérisé par une saison sèche de 7 mois étalée de Novembre à Mai et une saison pluvieuse de 5 mois (Juin à Octobre) marquée par un vent humide qu'est la mousson. Ce climat a une influence prépondérante sur le comportement des cultures à travers surtout la pluviosité et la température. Pendant la durée du test (mai à aout 2014), les données climatiques suivantes ont été enregistrées. pluies entrainent une dispersion de l'argile, provoquant ainsi une perte par lixiviation de ces éléments. En plus, cette pauvreté des sols en MO réduit énormément la Capacité d'Echange Cationique (CEC). Ce qui limite la capacité de rétention des ions $\mathrm{K}^{+}$, $\mathrm{Ca}^{2+}$ et $\mathrm{Mg}^{2+}$. Cet état des faits pose des problèmes techniques surtout aux petits producteurs à faibles revenus qui se doivent de gérer efficacement de faibles quantités d'engrais pour en tirer de meilleurs profits. Le fractionnement des engrais n'est-il pas une des clés de cette gestion ? Le présent travail cherche à évaluer l'effet des apports fractionnés des engrais minéraux sur les variables majeurs de croissance, de développement, de précocité et de productivité de la tomate en culture d'hivernage au centre du Burkina Faso. Il s'agit de déterminer la bonne fréquence d'apport des engrais minéraux sur la croissance et la productivité des variétés de tomate en culture d'hivernage.

L'évolution de la température au moment de la conduite de l'essai (mai à août 2014) est représentée sur la Figure 1 (a). Les températures moyennes mensuelles ont été de $32,9^{\circ} \mathrm{C}$ aux semis des tomates (mai) et à la fructification (juillet-aout) entre $29,8^{\circ} \mathrm{C}$ et $28,1^{\circ} \mathrm{C}$. La présente campagne a enregistré des pluies précoces depuis le mois d'avril et a suivie une évolution plus ou moins normale depuis mai jusqu'en août 2014 (Figure 1 (b)). Au total, 708,0 mm d'eau ont été enregistrées au cours du cycle végétatif de la tomate (mai-aout).

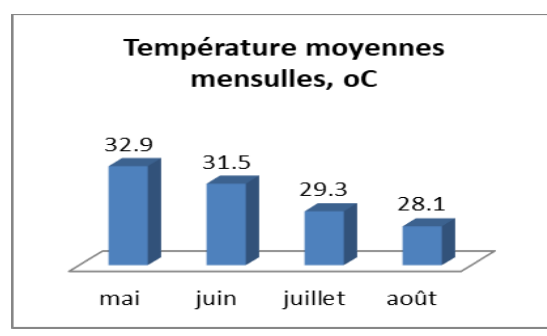

(a)

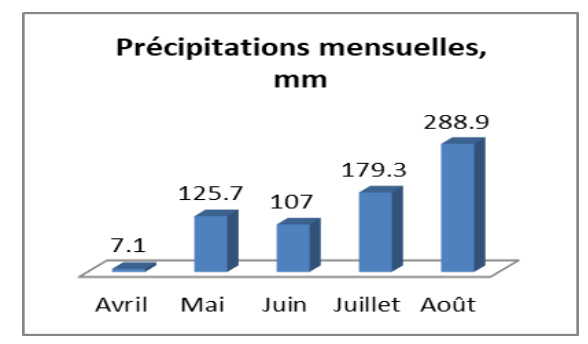

(b)

Figure 1: Températures moyennes mensuelles, campagne 2014/2015, site de Kamboinsé (a) et précipitations mensuelles, campagne 2014/2015, site de Kamboinsé (b) 
Le mois d'aout a été le plus arrosé avec 288,9 mm de précipitations. Comparez aux données climatiques annuelles moyennes enregistrées dans la zone de Ouagadougou pour la même période de culture (précipitation annuelle 788 $\mathrm{mm}$, température moyenne annuelle $29,1^{\circ} \mathrm{C}$ ), la période de végétation de la tomate a été très chaude (en moyenne $30,5^{\circ} \mathrm{C}$ ) et très humide. Ces conditions climatiques non idéales vont avoir un impact négatif sur les paramètres de productivité et au delà sur la qualité général des tomates récoltés. En effet, la tomate est une plante des régions chaudes dont le développement optimum requiert des températures comprises entre $21^{\circ} \mathrm{C}$ et $24^{\circ} \mathrm{C}$ (Naika et al, 2005). Les besoins en eau de la tomate pour son développement sont de 750 mm en 110 jours (MAHRH, 2007). Les sols de Kamboinsé sont classés comme des sols ferrugineux tropicaux lessivés reposant sur de matériaux sableux profonds et des sols hydromorphes peu humifères à pseudogley hérités en association avec des lithosols sur cuirasse ferrugineuse (KALOGA, 1969). Selon Boulet (1976), ces sols présentent une texture à dominance sablo argileuse en surface et argileuse en profondeur. La profondeur de la zone d'enracinement est très variable et peutêtre limitée par l'horizon induré (carapace ou cuirasse ferrugineuse).

\subsection{Matériel expérimental :} Trois variétés de tomate améliorée sont été utilisées dans notre étude. Il s'agit des variétés Padma (V1), Thorgal (V2) et Tomy (V3) à croissance déterminée. Les caractéristiques essentielles des variétés sont présentées dans le Tableau 1. L'essai a été conduit en pots sous abri «insect proof». pots utilisés sont des seaux en plastique de 6 litres. En moyenne, 4,5 kg de terre ont été mis dans chaque pot pour la conduite de l'essai. Les engrais minéraux utilisés dans cette étude étaient le NPK (14-23-14-6S) et l'Urée $(46 \% \mathrm{~N})$.

Tableau 1 : Caractéristiques essentielles des variétés

\begin{tabular}{|c|c|c|c|c|c|}
\hline Variétés & Origine & Précocité & $\begin{array}{l}\text { Forme } \\
\text { du fruit }\end{array}$ & $\begin{array}{l}\text { Poids } \\
\text { moyen fruit }\end{array}$ & Autres caractéristiques essentielles \\
\hline $\begin{array}{l}\text { Padma } \\
\text { F1 } \\
\text { (V1) }\end{array}$ & $\begin{array}{l}\text { East West } \\
\text { Seed } \\
\text { International } \\
\text { (Thailande) }\end{array}$ & $\begin{array}{l}\text { 1ère récolte } \\
60-70 \\
\text { JAR }\end{array}$ & $\begin{array}{l}\text { forme } \\
\text { ovale }\end{array}$ & 120 à $130 \mathrm{~g}$ & $\begin{array}{l}\text { Plant vigoureux; culture en saison } \\
\text { chaude et à l'hivernage; fruit de bonne } \\
\text { fermeté; résistance au virus de la } \\
\text { mosaïque de la tomate; tolérance au } \\
\text { flétrissement bactérien au Fusarium } \\
\text { oxysporum (races } 0 \text { et } 1 \text { ) }\end{array}$ \\
\hline $\begin{array}{l}\text { Thorgal } \\
\text { F1 } \\
\text { (V2) }\end{array}$ & $\begin{array}{l}\text { Technisem } \\
\text { (Français) }\end{array}$ & $\begin{array}{l}\text { 1 ̀̀re récolte } \\
65 \mathrm{JAR}\end{array}$ & $\begin{array}{l}\text { Forme } \\
\text { Ronde } \\
\text { légèreme } \\
\text { nt } \\
\text { aplatie }\end{array}$ & 120 à $130 \mathrm{~g}$ & $\begin{array}{l}\text { Culture en saison chaude et pendant } \\
\text { l'hivernage; fruit rouge vif; bonne } \\
\text { fermeté; tolérance au flétrissement } \\
\text { bactérien résistant au TMV (0) et au } \\
\text { Fusarium oxysporum f. sp. lycopersicum } \\
\text { (fol.0) }\end{array}$ \\
\hline $\begin{array}{l}\text { Tomy F1 } \\
\text { (V3) }\end{array}$ & USA & $\begin{array}{l}\text { 1ère récolte } \\
80 \text { à } 90 \\
\text { JAR }\end{array}$ & $\begin{array}{l}\text { Forme } \\
\text { ronde à } \\
\text { ovale }\end{array}$ & 70 à $80 \mathrm{~g}$ & $\begin{array}{l}\text { Culture en saison chaude et pendant } \\
\text { l'hivernage; très bonne fermeté } \\
\text { supporte mieux le transport et le } \\
\text { stockage; tolérance au flétrissement } \\
\text { bactérien résistant au TMV (0) et au } \\
\text { Fusarium oxysporum f. sp. lycopersicum } \\
\text { (fol.0) }\end{array}$ \\
\hline
\end{tabular}

JAR = Jour Après Repiquage 
3.3 Dispositif expérimental : L'essai a été installé suivant un dispositif split splot avec quatre répétitions. Les traitements (fréquences d'apport des engrais) étaient placés en parcelles principales et les variétés (trois) en parcelles secondaires.

Les doses de NPK et d'Urée appliquées étaient respectivement de $450 \mathrm{~kg} / \mathrm{ha}$ et de $200 \mathrm{~kg} / \mathrm{ha}$ :

- $\quad$ F1: Apport de 3 grammes de NPK et de 1,33 grammes d'urée par pot chaque deux (02) semaines à partir de 14 jours après levée.

- $\quad$ F2: Apport de 4,5 grammes de NPK et de 2 grammes d'urée par pot chaque trois (03) semaines à partir de 14 jours après levée.

- F3: Apport unique de 18 grammes de NPK par pot 14 jours après levée et 8 grammes d'urée par pot en deux fractions (trois semaines après levée et 6 semaines après levée).

\begin{tabular}{|c|c|c|}
\multicolumn{1}{c}{ F2 } & F3 & F1 \\
\hline V2 & V2 & V3 \\
\hline V1 & V3 & V2 \\
\hline V3 & V1 & V1 \\
\hline
\end{tabular}

Répétition 1

\begin{tabular}{|c|c|c|}
\multicolumn{1}{c}{ F3 } & F1 & F2 \\
\hline V3 & V2 & V1 \\
\hline V1 & V3 & V2 \\
\hline V2 & V1 & V3 \\
\hline
\end{tabular}

Répétition 2
Figure 2 : Schéma du dispositif expérimental

3.4 Conduite de l'essai : L'essai a commencé avec la stérilisation de la terre le 28 avril 2014. La terre a été stérilisée avec un autoclave à $120^{\circ} \mathrm{C}$. A cette température, tous les micro- organismes et les parasites ont été détruits. $1065,7 \mathrm{~kg}$ de cette terre stérilisée ont été mélangés avec 230,4 kg de fumier de bovin bien décomposé. La quantité du fumier a été calculée sur la base de 20 tonnes de fumier par ha. En milieu paysan, il est recommandé 25000 pieds de tomate par ha, cela signifie qu'un pied bénéficie de $0,8 \mathrm{~kg}$ de fumier soit 230,4 $\mathrm{kg}$ pour 288 plants. Quant à la quantité de la terre, elle a été calculée en fonction de la capacité des pots. Chaque pot ne peut contenir que $4,5 \mathrm{~kg}$ de terre, s'il est rempli en laissant $2 \mathrm{~cm}$ de la terre au bord du seau. De ces 4,5 kg, en déduisant les $0,8 \mathrm{~kg}$ de fumier, il fallait 3,7 $\mathrm{kg}$ de terre par pot soit $1065,7 \mathrm{~kg}$ de terre pour les 288 pots. Les pots ont été troués (05 trous par pot) avant de
Dans chaque répétition, les interactions entre les facteurs ont donné 9 parcelles élémentaires; d'où 36 parcelles élémentaires pour les 4 répétitions qui constituent l'essai. Chaque parcelle élémentaire est constituée de 8 pots soit 72 pots pour chaque répétition et 288 pots pour tout l'essai. Dans chaque répétition, les traitements principaux (fréquence d'apport) ont été randomisés et à l'intérieur de chaque traitement principal, les traitements secondaires (variétés) ont été également randomisés donnant le dispositif final ci- après (Figure 2: Schéma du dispositif expérimental). Les plants étaient espacés de $20 \mathrm{~cm}$ sur les lignes et les interlignes étaient de $120 \mathrm{~cm}$, soit une densité de plant de 41667 ha.

\begin{tabular}{|c|c|c|}
\multicolumn{1}{c|}{ F2 } & F3 & F1 \\
\hline V1 & V2 & V1 \\
\hline V2 & V3 & V2 \\
\hline V3 & V1 & V3 \\
\hline
\end{tabular}

Répétition 3

\begin{tabular}{|c|c|c|}
\multicolumn{1}{c|}{ F3 } & F2 & F1 \\
\hline V2 & V3 & V3 \\
\hline V3 & V1 & V1 \\
\hline V1 & V2 & V2 \\
\hline
\end{tabular}

Répétition 4 recevoir le mélange terre/ fumier. Ce mélange a été arrosé pendant une semaine pour accélérer la reprise de l'activité microbienne avant les semis. Les semis ont été réalisés le 16 mai 2014 à une profondeur d'environ $2 \mathrm{~cm}$. Dans chaque pot, il a été semé 5 graines. $50 \%$ des levées ont été observées cinq (05) jours après semis, le 21 mai 2014. Une semaine après levée, il a été procédé au démariage, laissant un plant par pot. Ce dernier a été enfoncé jusqu'au deux premières feuilles pour permettre un bon enracinement. L'eau des pluies a été la principale source d'eau des plantes, mais des irrigations complémentaires étaient faites au besoin. Ces irrigations se faisaient manuellement, avec des arrosoirs. Les mauvaises herbes étaient systématiquement arrachées des pots dès leurs apparitions. Un binage est fait à chaque application d'engrais. L'application des engrais a été faite sur la base 
des quantités vulgarisées en milieu paysan c'està-dire $450 \mathrm{~kg}$ de $\mathrm{NPK} /$ ha et $200 \mathrm{Kg}$ d'urée/ha. Un ha porte 25000 pieds de tomate, cela suppose que chaque pied bénéficie de 18 grammes de NPK et de 8 grammes d'urée. La tomate a besoin d'une fertilisation minérale jusqu'à la fructification. Elle demande davantage de l'azote pendant cette période (PIP et COLEACP, 2011). Pour ce faire, nous avons étalé les différentes fréquences d'apports jusqu'à 90 jours après levée. Le Tableau 2 cidessous donne la situation du nombre d'apports et des quantités apportées par traitement. La mesure des quantités apportées a été faite avec une balance électronique. L'application a débuté deux semaines après levée c'est-à-dire le 04 juin 2014. Les différentes dates d'apports sont résumées dans le Tableau 2 ci-après. Comme l'essai a été conduit dans une serre, les applications insecticides ont été faites au besoin. Au total, six traitements ont été effectués dont cinq avec le produit systémique PACHA (composée d'Acétamipride $10 \mathrm{~g} / 1$ et de Lambda- cyhalothrine $15 \mathrm{~g} / \mathrm{l}$ ) et un avec le Décis (deltaméthrine 12,5g/l). Les traitements ont atteint ce nombre à cause d'une déchirure du filet de l'abri par un vent le 20 juin 2014.

Tableau 2 : Nombre d'apports, quantité par application et dates d'apport

\begin{tabular}{l|c|c|c|c}
\hline Traitement & $\begin{array}{c}\text { Type } \\
\text { d'engrais }\end{array}$ & $\begin{array}{c}\text { Nombre } \\
\text { d'apports }\end{array}$ & $\begin{array}{c}\text { Quantité par } \\
\text { application }\end{array}$ & Dates d'apports \\
\hline F1 (Apport toutes & NPK & $90 / 14=6$ fois & $18 / 6=3 \mathrm{~g}$ & $04 / 06 ; 18 / 06 ;$ \\
les 2 semaines) & Urée & $90 / 14=6$ fois & $8 / 6=1,33 \mathrm{~g}$ & $02 / 07 ; 16 / 07 ;$ \\
& NPK & $90 / 21=4$ fois & $18 / 4=4,5 \mathrm{~g}$ & $04 / 06 ; 25 / 06 ;$ \\
\hline F2 (Apport toutes & Urée & $90 / 21=4$ fois & $8 / 4=2 \mathrm{~g}$ & $16 / 07 ; 06 / 08$ \\
\hline les 3 semaines) & NPK & 1 fois & $18 \mathrm{~g}$ & $04 / 06$ \\
\hline F3 Apport & Urée & 2 fois & $8 / 2=4 \mathrm{~g}$ & $11 / 06 ; 02 / 07$ \\
conventionnel & &
\end{tabular}

\subsection{Observations et mesures : Les} mesures ont porté sur les variables de croissance (hauteur des plants et diamètre de la tige principale), de développement et de précocité (dates de floraison et de nouaison) et enfin sur les variables de productivité (nombre de fleurs, de grappes à fruit, de fruits récoltés par plant). Pour ce qui est des variables de croissance, elles ont été mesurées tous les 10 jours; il s'agit de la hauteur des plants et le diamètre des tiges et ce, depuis le $20^{\text {ème }} \mathrm{JAL}$ jusqu'au $75^{\text {ème }}$ JAL. Seule la dernière mesure a été faite 15 jours après l'avant dernière. La hauteur des plants a été mesurée avec une règle et le diamètre avec un pied à colis. Ces mesures ont permis de vérifier l'effet des fréquences d'apport des engrais minéraux sur la croissance des plants. Concernant les variables de développement et de précocité, il a été noté :
- $\quad$ la date du début de floraison et $50 \%$ de floraison ;

- la hauteur d'insertion du premier bouquet floral ;

- la date du début de nouaison et $50 \%$ de nouaison ;

- la date du début des récoltes et la date de la fin des récoltes.

Au niveau des variables de productivité, il a été question dans chaque traitement de: compter le nombre de bouquets floraux par plant, le nombre de grappes à fruits par plant

Toutes les données quantitatives ont fait l'objet d'une analyse de la variance à deux critères de classification: «blocs» et «variétés». La comparaison des moyennes a été faite selon la méthode du test de STUDENT (ppds) au seuil de $5 \%$. 


\section{RESULTATS}

4.1 Effet sur la hauteur des plants : La Figure 3 présente l'évolution de la hauteur des plants de tomate selon la fréquence d'apport des engrais. Elle montre que les plants issus d'un apport de NPK et d'urée toutes les trois semaines (F2) ont cru plus rapidement que ceux qui étaient sous F1 et F3. Cette différence des hauteurs des plants sous F2 a été plus visible à partir de 40 Jours Après Levée (JAL). Les hauteurs des plants issus de la F1 (apport toutes les deux semaines) et la F3 (apport unique) ont été presque similaires depuis la levée jusqu'à la fin de la croissance. Les Figures $4 \mathrm{a}$, $4 \mathrm{~b}$ et $4 \mathrm{c}$ montrent l'évolution des hauteurs des plants de chaque variété en fonction des fréquences d'apport. Pour la variété Padma (Figure 4a), ce sont les plants qui ont bénéficié de la fertilisation toutes les trois semaines (F2) qui avaient une croissance plus rapide. Ils sont suivis des plants qui étaient sous apport unique (F3). Sous F1, la hauteur limite des plants est atteinte à $50 \mathrm{JAL}$ alors que sous $\mathrm{F} 2$ et $\mathrm{F} 3$, la hauteur limite est plus ou moins atteinte une dizaine de jours plus tard.

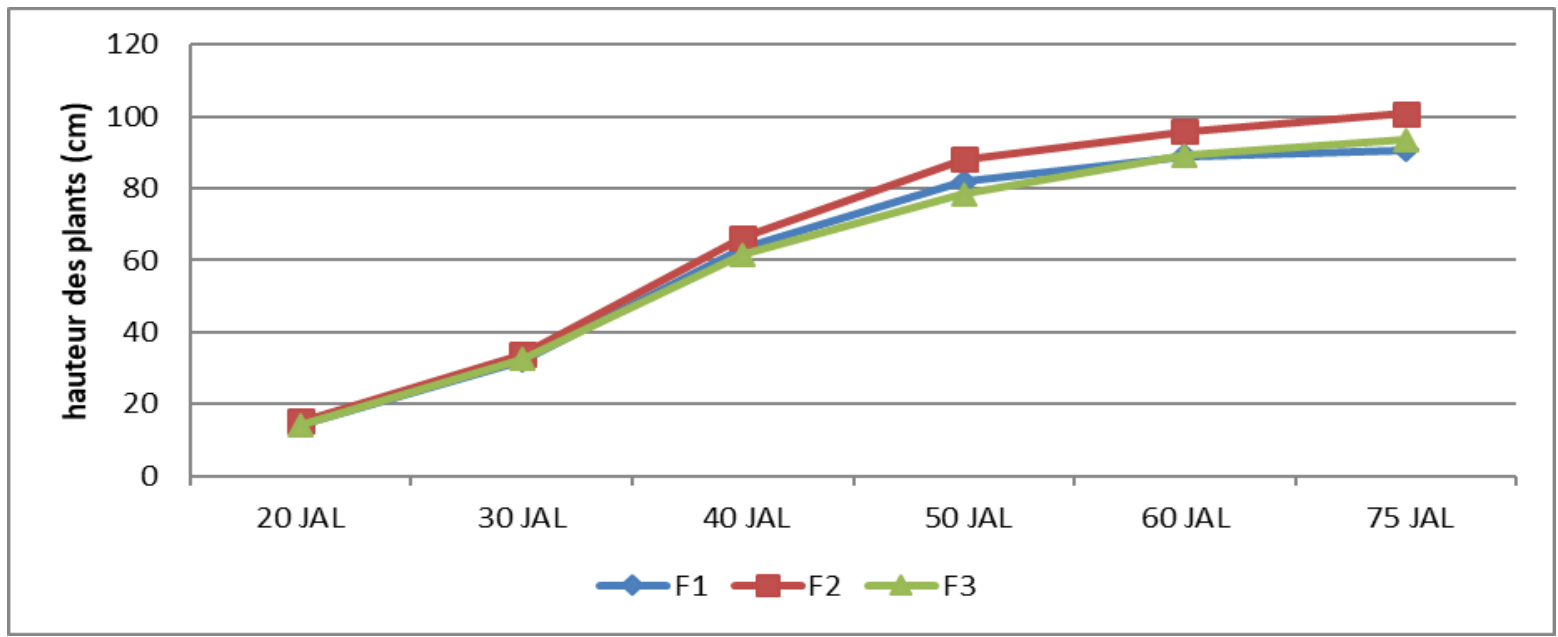

Figure 3 : Effet des fréquences d'apport des engrais sur la hauteur des plants.

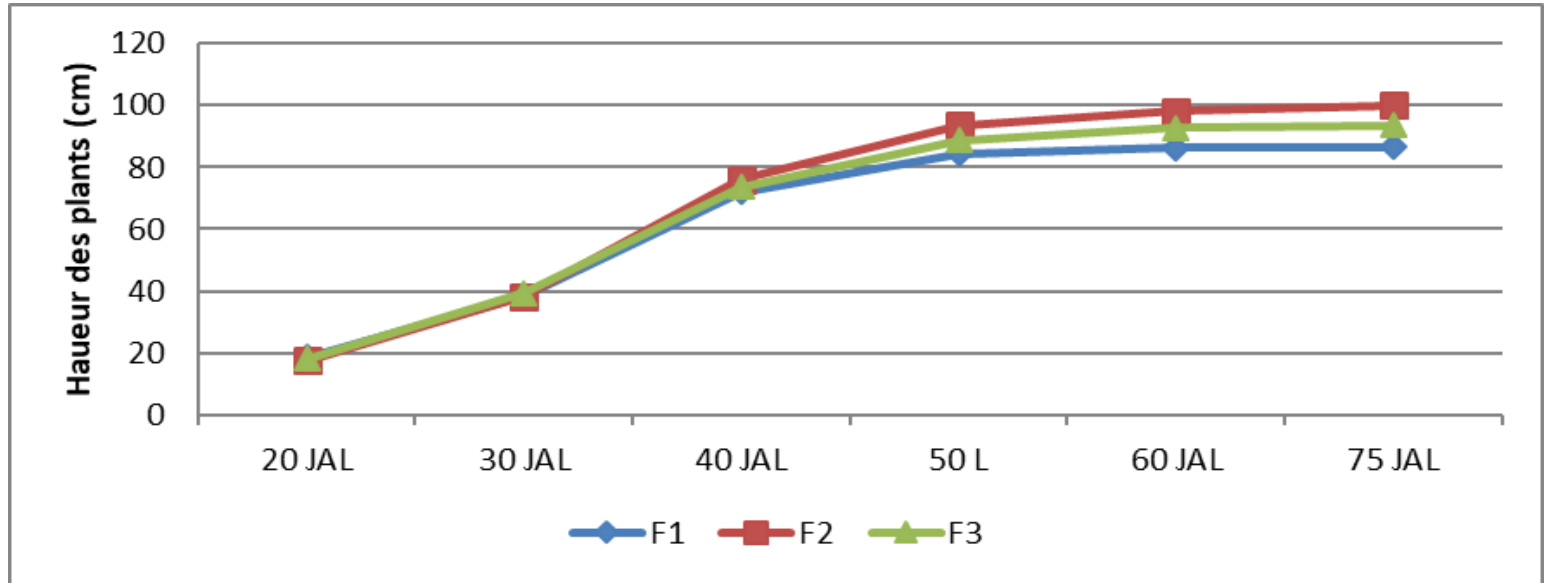

Figure 4a : Influence des fréquences d'apport sur la hauteur des plants de la variété Padma 


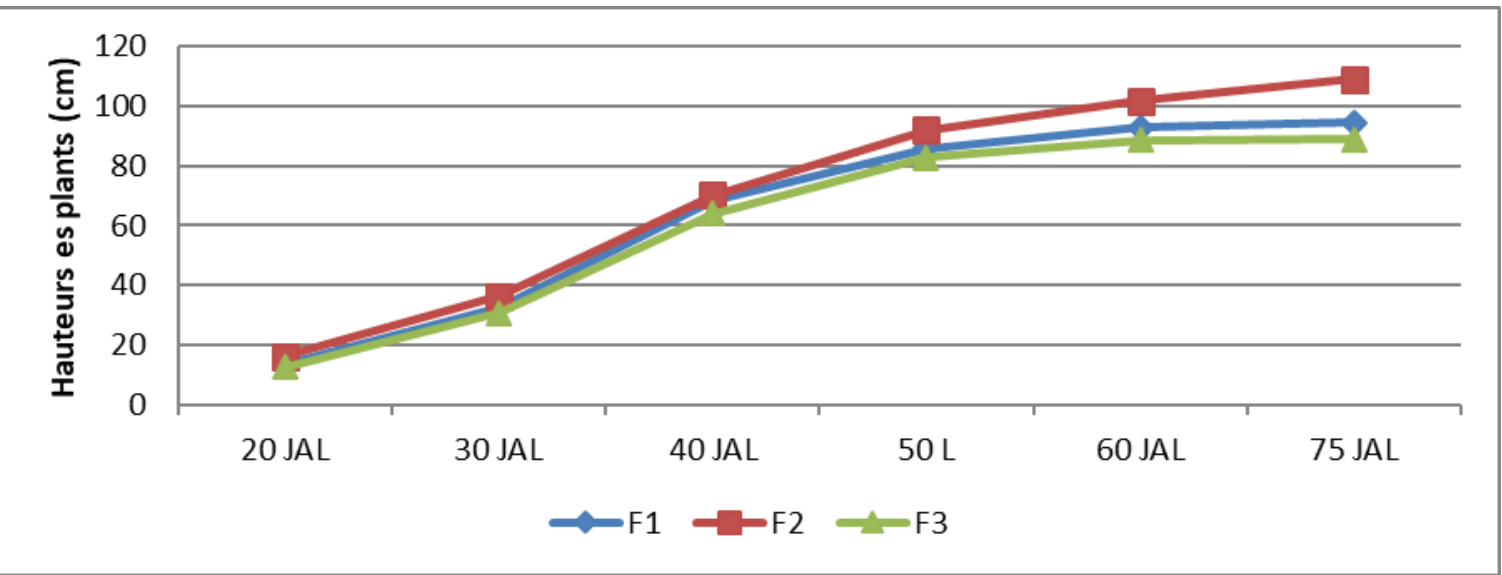

Figure 4b: Influence des fréquences d'apport sur la hauteur des plants de la variété Thorgal

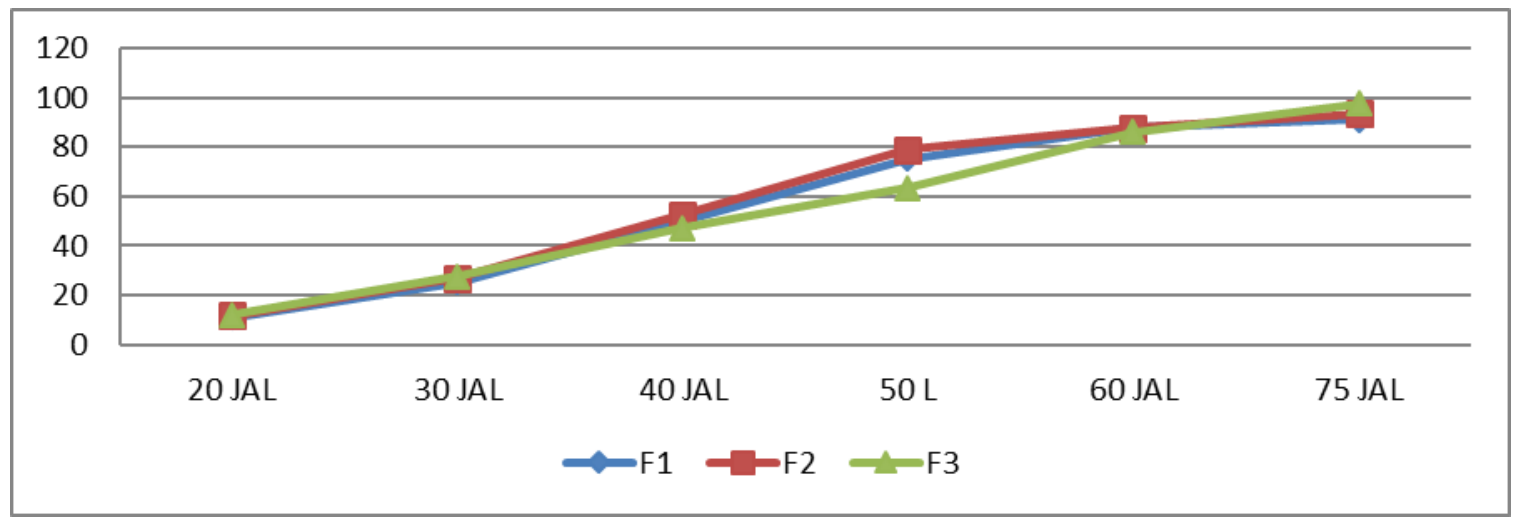

Figure 4c : Influence des fréquences d'apport sur la hauteur des plants de la variété Tomy

Pour la variété Thorgal (figure 4b), c'est également les plants qui ont été fertilisés toutes les trois semaines (F2) qui ont connu une croissance plus rapide. Ils sont suivis des plants qui ont bénéficié d'apport d'engrais toutes les deux semaines (F1). Sous F1 et F3, la hauteur limite est presque atteinte à $50 \mathrm{JAL}$ alors que sous F2, la croissance s'est poursuivie jusqu'à 75 JAL. Pour ce qui concerne la variété Tomy (Figure 4c), les plants sous fréquences d'apport $\mathrm{F} 1$ et F2 ont connu une croissance similaire. Leur croissance a été plus rapide que les plants sous apport unique (F3) jusqu'à $60 \mathrm{JAL}$.

\subsection{Effet de la fréquence de fertilisation} sur le diamètre de la tige principale : $\mathrm{La}$ Figure 5 présente l'évolution du diamètre de la tige principale selon la fréquence de fertilisation minérale. Les résultats indiquent une similarité pour toutes les trois fréquences d'apport des engrais depuis la levée jusqu'à 75 JAL. Les diamètres moyens maxima ont été de $9,3 \mathrm{~mm}$ pour la fréquence d'apport F1, 9,2 $\mathrm{mm}$ pour la fréquence d'apport $\mathrm{F} 2$ et $9,1 \mathrm{~mm}$ pour la fréquence de fertilisation F3. Le diamètre maximum de la tige a été atteint à partir du $60^{\text {ème }}$ JAL. 


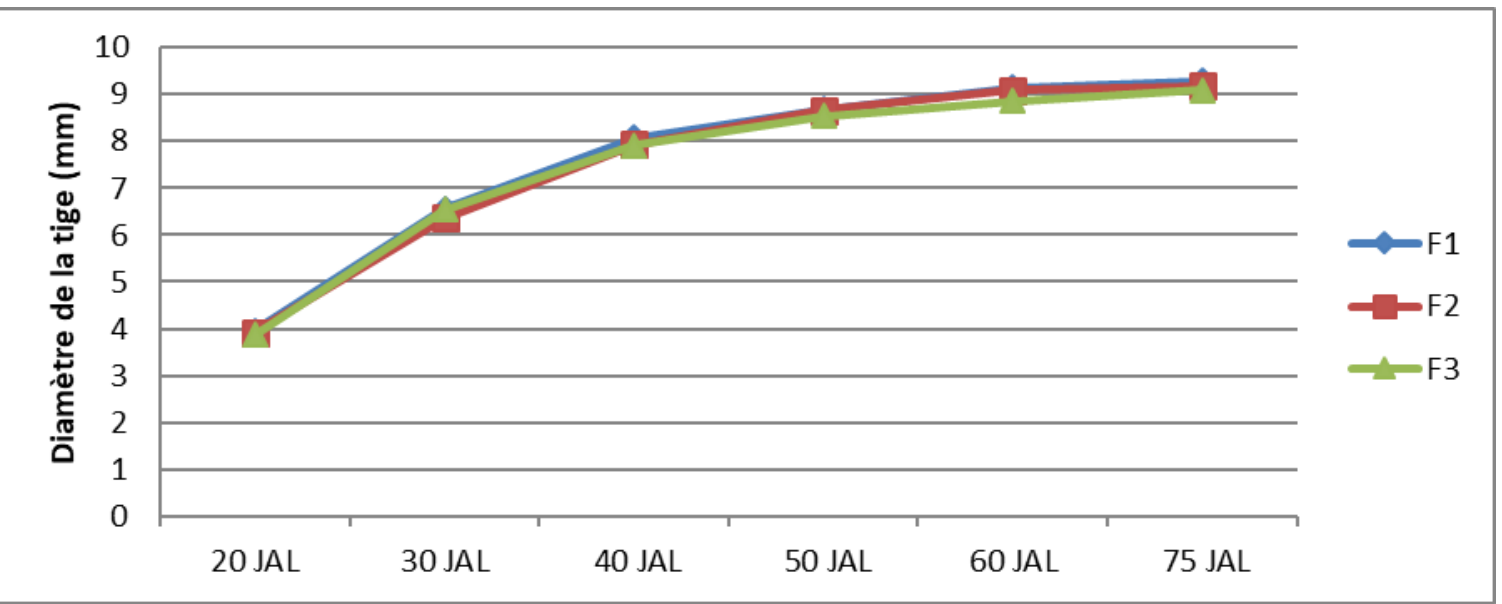

Figure 5: Effets des fréquences d'apports des engrais sur le diamètre de la tige de la tomate.

Les Figures $5 \mathrm{a}, 5 \mathrm{~b}$ et $5 \mathrm{c}$ présentent, par variété, l'évolution des diamètres de la tige principale sous les trois fréquences de fertilisation. Elles montrent une similarité des diamètres de la tige principale sous les trois fréquences d'apport pour chaque variété. Pour les variétés Padma et Thorgal, le diamètre maximal est plus ou moins atteint à $50 \mathrm{JAL}$ alors que pour Tomy, la croissance s'est poursuivie jusqu'à 75 JAL.

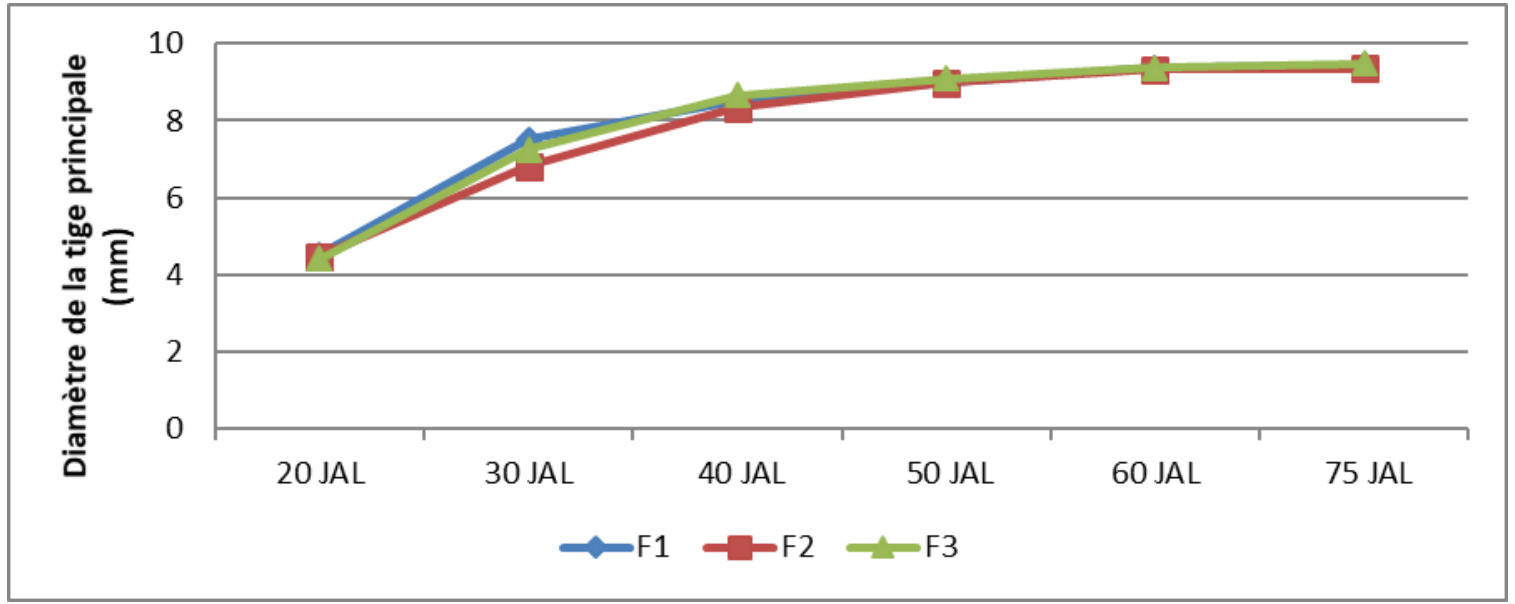

Figure 5a: Effets des fréquences d'apports sur le diamètre de la tige des plants de Padma 


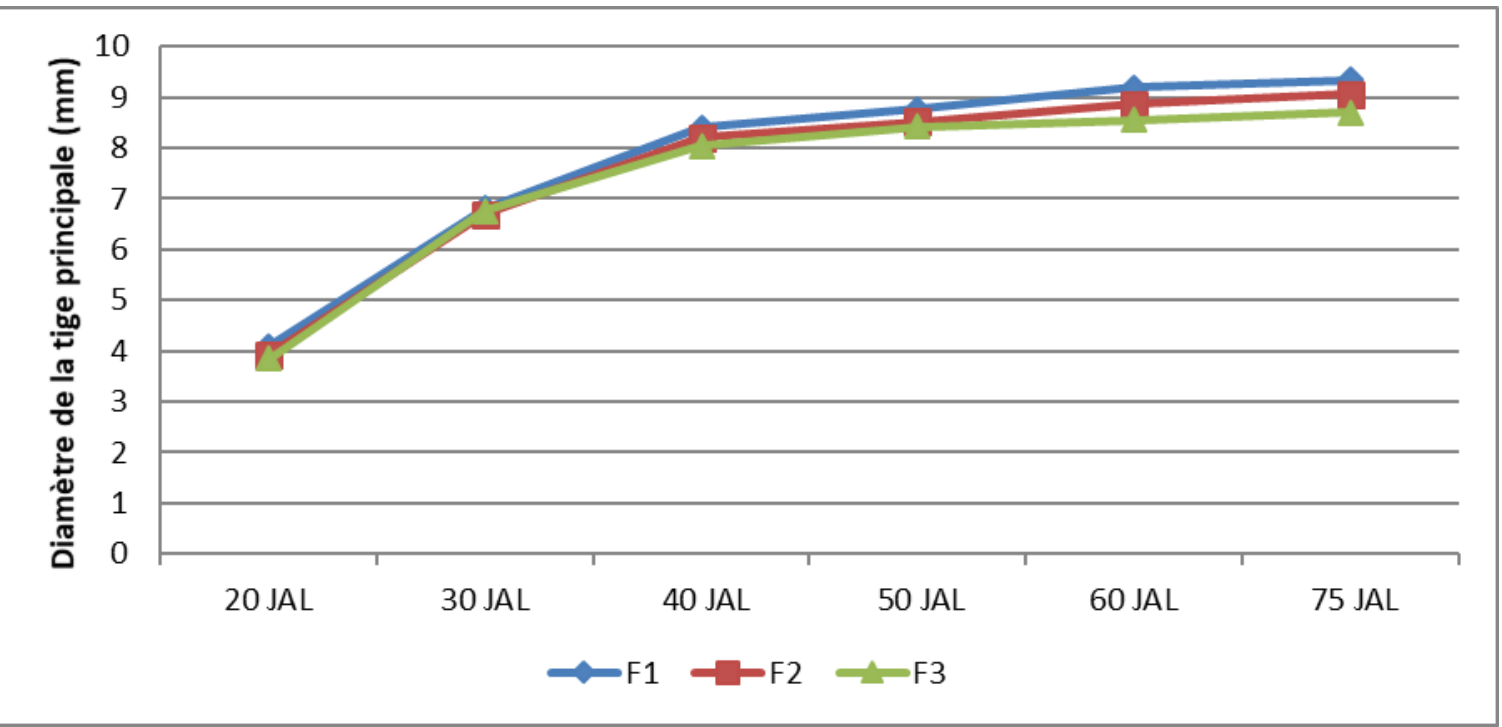

Figure 5b: Effets des fréquences d'apports sur le diamètre de la tige des plants de Thorgal

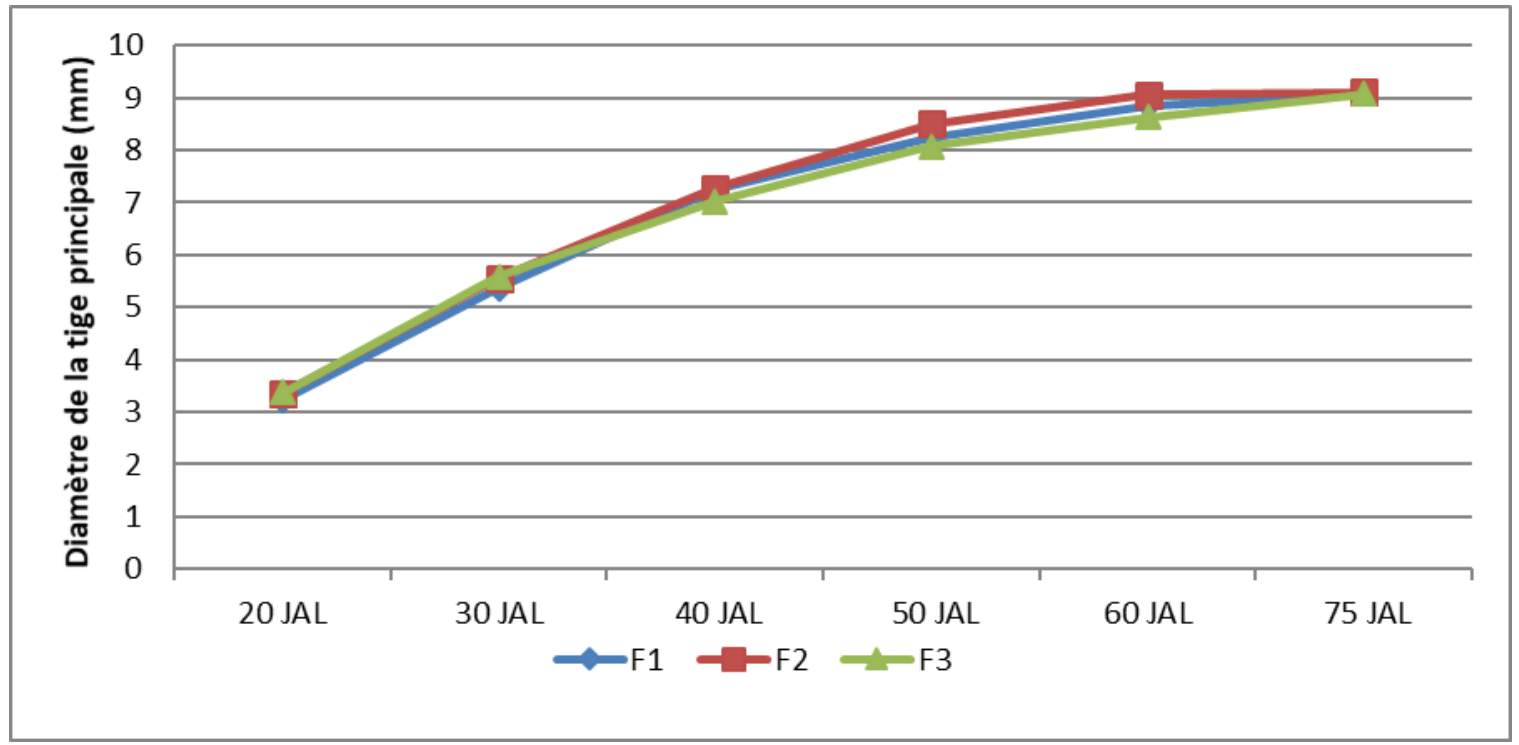

Figure 5c : Influence des fréquences d'apport sur le diamètre de la tige des plants de Tomy

\subsection{Effet de la fréquence d'apport sur la} floraison: A la Figure 6 est présentée la floraison (début et $50 \%$ ) des plants de tomate sous les trois fréquences de fertilisation. Il est constaté que sous le traitement F3 (apport conventionnel des engrais), le début de la floraison est retardé d'un jour par rapport aux plants sous F1 et F2. Par contre, tous les traitements avaient le même nombre de jours pour la floraison à 50\%. Les variétés Padma et Thorgal ont fleuri plus tôt que la variété Tomy dans toutes les fréquences d'apport (Tableau 3). Elles ont atteint également $50 \%$ de floraison pour toutes fréquences de fertilisation avant la variété Tomy. 
Tableau 3 : Influence des fréquences d'apport sur la précocité de floraison (nombre de JAL)

\begin{tabular}{|c|c|c|c|c|c|c|c|}
\hline Traitements & & \multicolumn{3}{|c|}{ Début de floraison } & \multicolumn{3}{|c|}{$50 \%$ de floraison } \\
\hline & & Padma & Thorgal & Tomy & Padma & Thorgal & Tomy \\
\hline F1 & & $32 \mathrm{~b}$ & $32 \mathrm{~b}$ & $38 a$ & $35 b$ & $36 \mathrm{~b}$ & $40 \mathrm{a}$ \\
\hline$\overline{\mathrm{F}} 2$ & & $33 \mathrm{~b}$ & $33 \mathrm{~b}$ & $37 a$ & $36 \mathrm{~b}$ & $36 b$ & $41 \mathrm{a}$ \\
\hline F3 & & $33 \mathrm{~b}$ & $33 \mathrm{~b}$ & $38 \mathrm{a}$ & $35 b$ & $36 \mathrm{~b}$ & $40 \mathrm{a}$ \\
\hline \multirow{4}{*}{$\begin{array}{l}\text { Source de } \\
\text { variation } \\
\text { (test F) }\end{array}$} & Répétition & \multicolumn{3}{|c|}{$\mathrm{P}=0,39$} & \multicolumn{3}{|c|}{$\mathrm{P}=0,28$} \\
\hline & Fertilisation & \multicolumn{3}{|c|}{$\mathrm{P}=0,29$} & \multicolumn{3}{|c|}{$\mathrm{P}=0,51$} \\
\hline & Variété & \multicolumn{3}{|c|}{$\mathrm{P}<0,001$} & \multicolumn{3}{|c|}{$\mathrm{P}<0,001$} \\
\hline & Fertilisation*Variété & \multicolumn{3}{|c|}{$\mathrm{P}=0,19$} & \multicolumn{3}{|c|}{$\mathrm{P}=0,96$} \\
\hline
\end{tabular}

NB : Les moyennes d'une même colonne affectées de la même lettre ne sont pas significativement différentes au seuil de $5 \%$ selon le test de la LSD. $\mathrm{P}<0,05=$ Variation significative $; \mathrm{P}<0,01=$ Variation très significative $; \mathrm{P}<0,001=$ variation hautement

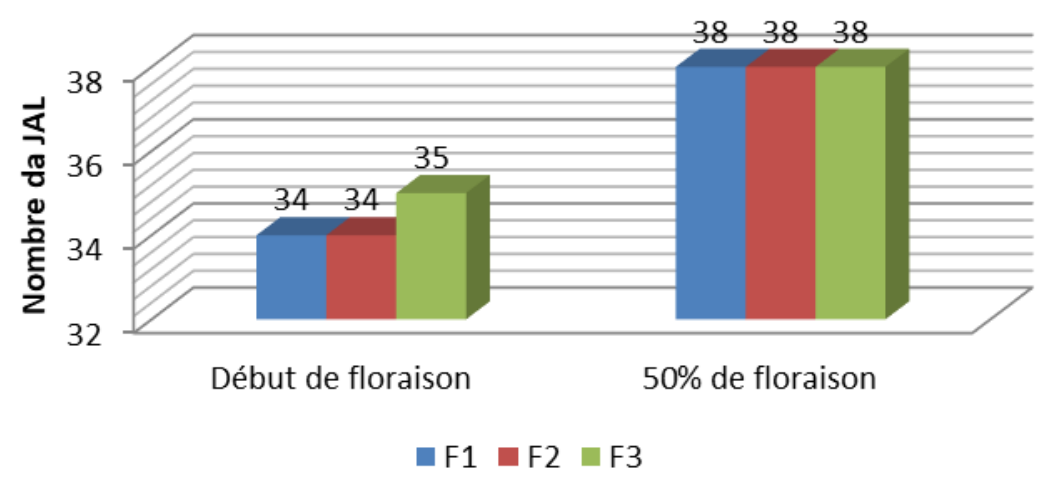

Figure 6 : Effet des fréquences d'apport des engrais sur la floraison

4.4 Effet de la fréquence d'apport sur la hauteur d'insertion du 1er bouquet floral: La figure 7 montre le niveau d'insertion du $1 \mathrm{er}$ bouquet floral sur les plants de la tomate sous les trois fréquences d'apport des engrais. Il ressort que la hauteur d'insertion du 1er bouquet floral est plus basse sur les plants sous apport conventionnel (F3) par rapport aux autres fréquences de fertilisation F1 et F2. Le 1er bouquet floral sous F1 était aussi plus bas que celui apparu sous F2.

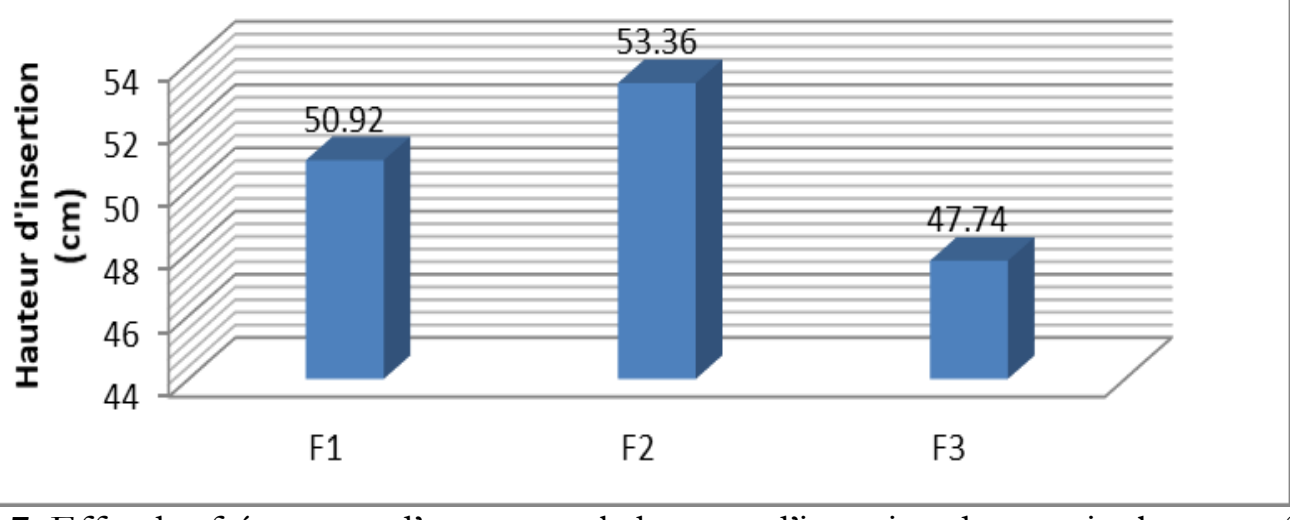

Figure 7: Effet des fréquences d'apport sur la hauteur d'insertion du premier bouquet floral 
La figure 8 montre que quelle que soit la fréquence de fertilisation, la hauteur d'insertion $\mathrm{du} 1^{\text {er }}$ bouquet floral est plus élevée chez la variété Padma que chez les deux autres variétés.
Pour l'ensemble des variétés, l'apport des engrais toutes trois semaines a induit une hauteur d'insertion plus élevé.

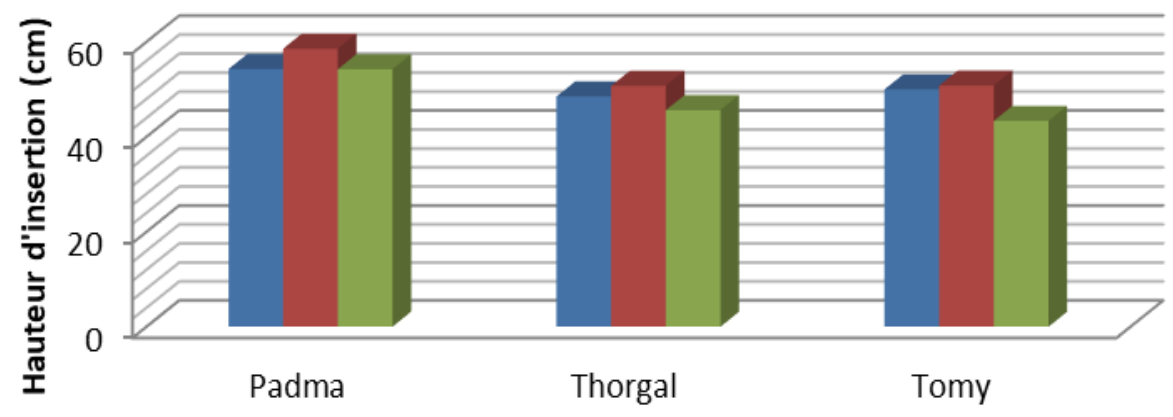

$\mathrm{F} 1 \square \mathrm{F} 2 \square \mathrm{F} 3$

Figure 8: influence des fréquences d'apport sur la hauteur d'insertion du ${ }^{\text {er }}$ bouquet floral

4.5 Effet de la fréquence d'apport sur la nouaison des variétés: La figure 9 présente l'influence des fréquences d'apport des engrais sur le début et les $50 \%$ de nouaison de la tomate. Les résultats montrent que sous l'apport unique (F3) et sous l'apport toutes les deux semaines (F1) d'engrais NPK et d'Urée, la nouaison a commencé un peu plus tôt que sous apport toutes les trois semaines. Selon la variété
(Tableau 4), la nouaison a commencé plus tôt pour les variétés Padma et Thorgal pour toutes les fréquences d'apport. Elle a été tardive au niveau de la variété Tomy. Les plants de la variété Tomy bénéficiant de la fréquence d'apport F1 ont débuté la nouaison plus tôt que les plants issus des fréquences d'apport F2 et F3.

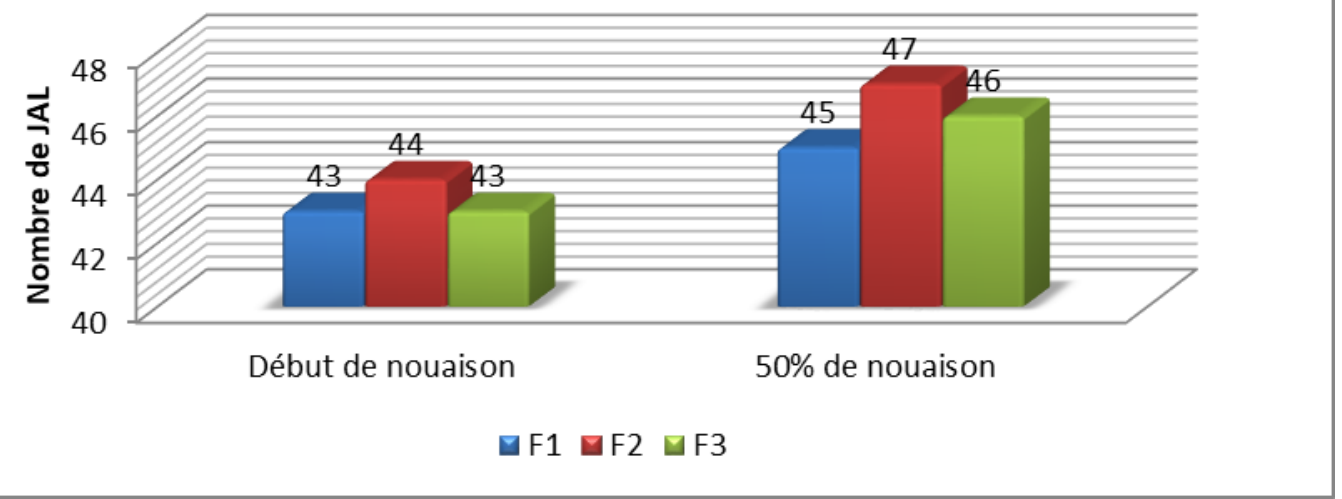

Figure 9: Effet des fréquences d'apport des engrais sur la nouaison

4.6 Effet de la fréquence d'apport sur les nombres de bouquets floraux et de grappes à fruits: Le tableau 5 présente le nombre de bouquets floraux par plant et le nombre de grappes à fruits par plant. Il n'y a pas eu de différences significatives entre les trois traitements pour le nombre de bouquets floraux et de grappes à fruits. Il est constaté que sous toutes les fréquences d'apport, 75\% des bouquets floraux de la variété Padma ont été 
transformés en grappes à fruits. Pour la variété Thorgal, 46 à 50\% des bouquets floraux sont devenus des grappes à fruits sous toutes les formes de fertilisation. $67 \%$ des bouquets floraux de la variété Tomy sous F1 et F2 ont été transformés en grappes à fruits alors que sous $\mathrm{F} 3$, seulement $50 \%$ des bouquets floraux se sont transformés en grappes à fruits.

Tableau 5: Effet des fréquences d'apport sur les nombres de bouquets floraux et de grappes à fruit

\begin{tabular}{|c|c|c|c|c|c|c|c|}
\hline \multirow[t]{2}{*}{ Traitements } & & \multicolumn{3}{|c|}{$\begin{array}{l}\text { Nombre de bouquets } \\
\text { floraux par plant }\end{array}$} & \multicolumn{3}{|c|}{$\begin{array}{l}\text { Nombre de grappes à fruits } \\
\text { par plant }\end{array}$} \\
\hline & & Padma & Thorgal & Tomy & Padma & Thorgal & Tomy \\
\hline $\begin{array}{l}\text { F1 } \\
\text { F2 } \\
\text { F3 } \\
\text { Source de } \\
\text { variation } \\
\text { (test F) }\end{array}$ & $\begin{array}{l}\text { Répétition } \\
\text { Fertilisation } \\
\text { Variété } \\
\text { Fertilisation*Variété }\end{array}$ & $\begin{array}{l}8 b \\
8 b \\
8 b\end{array}$ & $\begin{array}{c}10 \mathrm{a} \\
11 \mathrm{a} \\
10 \mathrm{a} \\
\mathrm{P}=0,85 \\
\mathrm{P}=0,41 \\
\mathrm{P}=0,009 \\
\mathrm{P}=0,66\end{array}$ & $\begin{array}{c}9 \mathrm{a} \\
9 \mathrm{a} \\
10 \mathrm{a}\end{array}$ & $\begin{array}{l}6 a \\
6 a \\
6 a\end{array}$ & $\begin{array}{c}5 \mathrm{~b} \\
5 \mathrm{~b} \\
5 \mathrm{~b} \\
\mathrm{P}=0,50 \\
\mathrm{P}=0,43 \\
\mathrm{P}=0,034 \\
\mathrm{P}=0,072\end{array}$ & $\begin{array}{l}6 a \\
6 a \\
5 b\end{array}$ \\
\hline
\end{tabular}

NB: Les moyennes d'une même colonne affectées de la même lettre ne sont pas significativement différentes au seuil de $5 \%$ selon le test de la LSD. $\mathrm{P}<0,05=$ Variation significative $; \mathrm{P}<0,01=$ Variation très significative $; \mathrm{P}<0,001=$ variation hautement significative.

\subsection{Effet de la fréquence d'apport sur la} récolte : Sur la Figure 10 est présentée les dates de récolte (début et fin) des plants de la tomate sous les trois fréquences de fertilisation. Les résultats montrent que la récolte des plants sous apport F1 a débuté un et deux jours plus tôt que sous F3 et F2, respectivement. Par contre sur la fin de la récolte, tous les traitements ont fourni le même résultat. La récolte a été plus hâtive pour les variétés Padma et Thorgal quelle que soit la fréquence d'apport. La variété Tomy par contre, est plus tardive (Tableau 6). La fertilisation n'a pas eu l'effet sur la fin des récoltes.

Tableau 6 : Effets des fréquences d'apport sur la précocité des récoltes et la fin des récoltes

\begin{tabular}{l|c|c|c|c|c|c|c}
\hline Traitements & & \multicolumn{3}{|c|}{ Début des récoltes } & \multicolumn{3}{c}{ Fin des récoltes } \\
\hline & & Padma & Thorgal & Tomy & Padma & Thorgal & Tomy \\
\hline F1 & & $73 \mathrm{~b}$ & $72 \mathrm{~b}$ & $83 \mathrm{a}$ & $86 \mathrm{~b}$ & $86 \mathrm{~b}$ & $96 \mathrm{a}$ \\
F2 & $76 \mathrm{~b}$ & $73 \mathrm{~b}$ & $84 \mathrm{a}$ & $86 \mathrm{~b}$ & $86 \mathrm{~b}$ & $94 \mathrm{a}$ \\
F3 & & $73 \mathrm{~b}$ & $73 \mathrm{~b}$ & $86 \mathrm{a}$ & $86 \mathrm{~b}$ & $86 \mathrm{~b}$ & $96 \mathrm{a}$ \\
\hline Source de & Répétition & \multicolumn{3}{|c|}{$\mathrm{P}=0,098$} & \multicolumn{3}{|c}{$\mathrm{P}=0,43$} \\
variation & Fertilisation & \multicolumn{3}{|c|}{$\mathrm{P}=0,003$} & \multicolumn{3}{|c}{$\mathrm{P}=0,40$} \\
(test F) & Variété & \multicolumn{3}{|c|}{$\mathrm{P}<0,001$} & \multicolumn{3}{c}{$\mathrm{P}=0,001$} \\
& Fertilisation*Variété & \multicolumn{3}{c|}{$\mathrm{P}=0,003$} & & \multicolumn{3}{c}{$\mathrm{P}=0,44$} \\
\hline
\end{tabular}

NB : Les moyennes d'une même colonne affectées de la même lettre ne sont pas significativement différentes au seuil de $5 \%$ selon le test de la LSD. $\mathrm{P}<0,05=$ Variation significative $; \mathrm{P}<0,01=$ Variation très significative $; \mathrm{P}<0,001=$ variation hautement significative. 


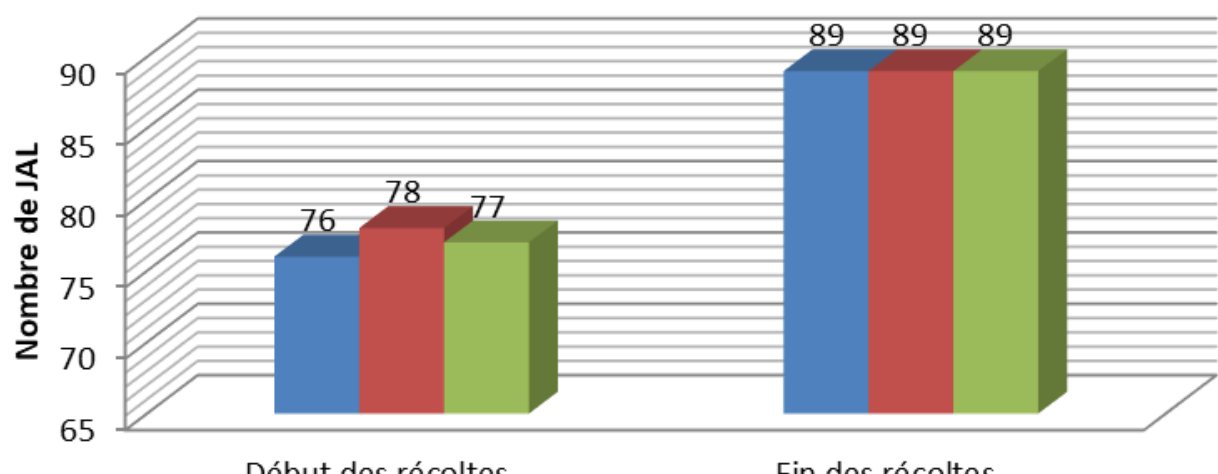

Début des récoltes

Fin des récoltes

F1 F2 $\square$ F3

Figure 10 : Effet des fréquences de fertilisation sur la précocité et la fin des récoltes

\section{$5 \quad$ DISCUSSION}

\subsection{Effets sur les variables de croissance} et de développement des tomates: Dans l'ensemble, s'agissant du comportement végétatif, les plants de toutes les variétés ont atteints des hauteurs plus ou moins supérieurs à leurs hauteurs moyennes en culture sans abri. En effet, à $75 \mathrm{JAL}$, les hauteurs des plants étaient comprisses entre 93 et $97 \mathrm{~cm}$ alors que Marques et Moreau (2007) ont montré que les plantes de tomate à croissance déterminée ne peuvent dépasser une hauteur de 60 à $80 \mathrm{~cm}$ selon les variétés et les conditions du milieu. Ce comportement est certainement dû à l'effet des températures plus élevées sous abri et de la lumière diffuse sous abri. Nos résultats sont conformes avec ceux obtenus par Djidji et al (2010). Les travaux de Djidji et al (2010) ont prouvé que les plantes cultivées sous abri ont une taille supérieure à celle des plantes cultivées en plein champ. Selon eux, ce comportement semble lié à un phototropisme sous l'abri, où la lumière diffuse a tendance à attirer les plantes dans leur croissance, ce qui n'est pas le cas dans les conditions culturales en plein champ. Les courbes des hauteurs des plants (Figures 4 et 5) en fonction de la fréquence d'apport des engrais présentent une différenciation significative. Cette situation s'expliquerait par la disponibilité des engrais pour les plantes suite au fractionnement. En effet, comme l'ont montré différents auteurs, l'azote est l'élément le plus important pour la vie de la plante. Extrait du sol par toutes les plantes et de l'air par quelques plantes, il en est le moteur et sert à construire toutes les parties vertes qui assurent la croissance et la vie des plantes (Lafond, 2004 ; Galla Bi Trezi et al., 2011). La fréquence d'apport F2 a eu un impact plus significatif sur la hauteur des plants que les fréquences d'apport F1 et la F3 qui ont donné des plants identiques en taille. Il nous semble que la fréquence d'apport F2 et les quantités apportées conduisent à une végétation abondante certainement en rendant suffisamment disponible les éléments nutritifs au moment où la plante en a besoin. Les quantités apportées à cette fréquence d'apport au début de la fertilisation ont peut-être permis la création d'un équilibre ionique du milieu favorable à une bonne absorption des plantes. La supériorité en taille des plantes des interactions F2*Padma et F2*Thorgal (Figure 5b) soutient cette assertion. Selon Abdellatif et al. (2010), pour un bon développement et une bonne production de la plante de tomate, il faut un équilibre ionique selon les différents stades de la croissance. Une étude menée par ces derniers sur une culture de tomate hors sol a permis de déterminer des équilibres ioniques de la solution nutritive. Selon les résultats de cette étude, la 
concentration en $\mathrm{N}$ du milieu nutritionnel de la plante doit baisser au fur et à mesure de la plantation à la fin des récoltes passant de $220 \mathrm{mg} / 1$ à $100 \mathrm{mg} / \mathrm{l}$. La concentration en $\mathrm{P}_{2} \mathrm{O}_{5}$ qui suit la même évolution que $\mathrm{N}$ doit être toujours $65 \%$ de la concentration de ce dernier. Par contre la concentration en $\mathrm{K}_{2} \mathrm{O}$ doit augmenter passant de $120 \%$ de la concentration de $\mathrm{N}$ au moment de la plantation à $200 \%$ de $\mathrm{N}$ au début des récoltes pour retomber à 180\% de $\mathrm{N}$ à la fin des récoltes.

\subsection{Effets des fréquences d'apports la} floraison et la nouaison: Concernant la floraison et la nouaison (figures 8 et 11) la fréquence d'apport n'a pas eu d'effet sur ces facteurs. Cependant, la précocité de la récolte et la hauteur d'insertion du $1^{\text {er }}$ bouquet floral semblent être influencées par la fréquence d'apport des engrais minéraux. L'absence d'effet significatif de la fréquence d'apport des engrais sur la date de floraison et la date de nouaison révèle dans nos conditions expérimentales que ces deux variables sont liées à des caractères génétiques. Les effets de la fréquence d'apport seraient masqués par l'influence du patrimoine génétique. Djidji et al. (2010) ont obtenus les mêmes résultats sur la tomate en culture sous abri. Ces auteurs ont montré que la date de floraison est un caractère génétique intrinsèque, propre à la variété. Elle est donc peu influencée par les facteurs extérieurs. Contrairement aux précédentes variables, un effet significatif de la fréquence d'apport a été observé sur le début des récoltes et la hauteur d'insertion du $1^{\text {er }}$ bouquet floral (figures 9, 10 et 12). Il semble que la hauteur d'insertion du $1^{\text {er }}$ bouquet floral soit liée au génotype (Nyembo et al., 2013). Dans ce cas, la taille des plantes qui a été proportionnelle aux fréquences d'apport pourrait expliquer cette différence observée dans la hauteur d'insertion $\mathrm{du} 1^{\text {er }}$ bouquet floral. Les apports rapprochés (toutes les deux ou trois semaines du NPK et de l'Urée) permettent une disponibilité accrue de l'azote surtout qui est un élément de croissance des plants. Il ya eu peut être un effet azote sur l'élongation des cellules de la tige, ce qui pourrait justifier le niveau d'insertion plus élevée du premier bouquet floral que l'apport conventionnel.

5.3 Effet de la fréquence d'apports sur les nombres de bouquets floraux et de grappes de fruits : Il n'y a pas eu de différences significatives entre les trois traitements pour le nombre de bouquets floraux et de grappes à fruits. Ce paramètre a été beaucoup soumis aux influences extérieures notamment par les températures qui ont été très défavorables au cours de la période végétative des tomates. En effet, la température moyenne de $30^{\circ} \mathrm{C}$ enregistrée à l'extérieur au cours des mois de mai et de juin a été à l'intérieur de la serre plus élevée. Or la tomate est très exigeante en température et redoute les fortes températures (Polese, 2007). A cet effet, Shankara (2005), souligne pour la plupart des variétés des températures optimales entre 21 et $24^{\circ} \mathrm{C}$. Par exemple, Chougar (2011) signale au Maroc pour la plupart des variétés des températures optimales de $18^{\circ} \mathrm{C}$ le jour et de 15 à $25^{\circ} \mathrm{C}$ la nuit. Ces fortes températures pendant la période de fructification favorisent la croissance de la plante au détriment de l'inflorescence qui peut avorté (IAV, 1999). En outre, l'essai a été victime d'une attaque sévère de Tetranychus urticae communément appelé l'araignée rouge que nous n'avions pas pu contrôler. Elle a réduit la durée de la production en stoppant la floraison. Néanmoins comme tous les traitements étaient dans les mêmes conditions, les résultats sont comparables. Cependant on sait d'après Kolev (1976) que l'apport du phosphore sous une forme assimilable favorise la formation des fleurs et des fruits et augmente la précocité.

\subsection{Effet des fréquences d'apports sur le} nombre de récoltes: Selon Zuang (1984) et Tirilly et Bourgeois (1999), l'effet génotypique, la saison de culture, les conditions climatiques et le taux de nouaison influencent ce caractère. Par exemple, l'apport du phosphoresous une forme aisément assimilable d'après Thompson et Kelly (1957 in Kolev (1976), favorise la formation et le développement des fleurs et des 
fruits et augmente la précocité des tomates. Selon Jacob et Janssen (1979), l'excès d'azote favorise une végétation excessive au détriment de la fructification et diminue donc la précocité. Laumonnier (1979) ajoute que l'azote favorise un développement foliacé trop important et une maturité plus tardive. Pour le début des récoltes, les plants issus des fréquences d'apport F2 ont été les derniers à arriver à maturité. C'est aussi les plants de ce traitement F2 qui ont

\section{CONCLUSION}

Cette étude a consisté à vérifier les effets du fractionnement des engrais minéraux sur la croissance, et la productivité de la tomate en culture d'hivernage. Les résultats obtenus nous ont permis de tirer les conclusions suivantes:

- les plants issus d'un apport de NPK et d'urée toutes les trois semaines (F2) ont cru plus rapidement que ceux qui étaient sous $\mathrm{F} 1$ et $\mathrm{F} 3$;

- l'apport des engrais toutes trois semaines (F2) a induit une hauteur d'insertion plus élevé du 1er bouquet floral pour l'ensemble des variétés; - sous l'apport unique (F3) et l'apport toutes les deux semaines (F1) d'engrais NPK et d'Urée, la nouaison a commencé un peu plus tôt par rapport à la fréquence d'apport de toutes les trois semaines (F2);

- l'évolution des diamètres de la tige principale sous les trois fréquences de fertilisation a montré une similarité pour chaque variété ;

7 REFERENCES BIBLIOGRAPHIQUES

Abdellatif E.F. et Noureddine C., 2010. Etude de base sur la culture de la tomate au Maroc. Edit. FAO et ONSSA, 110p.

Bado B.V., 1994. Modification chimique d'un sol ferralitique sous l'effet de fertilisants minéraux et organiques: conséquences sur les rendements d'une culture continue de maïs, (1994) 57p

Boulet R., 1976. Notice des Cartes de ressources en sols de la Haute-Volta. Echelle /XXII 000. ORSTOM, Paris.

Bunasols (Bureau National des Sols) (1985) Etat de connaissance de la fertilité des sols du Burkina Faso. Documentation connu une croissance plus importante. Il nous semble que cette forme d'apport des engrais NPK et urée (F2) a permis une disponibilité importante de l'azote prolongeant ainsi la période de la végétation au détriment du développement des organes reproducteurs. Selon Abdellatif et al. (2010), une consommation importante en azote provoque une végétation abondante et un retard de maturation des fruits.

- il n'y a pas eu de différences significatives entre les trois traitements pour le nombre de bouquets floraux et de grappes à fruits formés. Toutefois, il serait nécessaire de poursuivre cette étude en mettant en place un essai en milieu paysan pour mieux prendre en compte l'effet de la fréquence d'apport sur les rendements et l'évolution phytosanitaire (maladies fongiques, bactériennes, virales). En outre, nous suggérons qu'une étude soit conduite pour évaluer la rentabilité économique de la fréquence d'apport des engrais minéraux car le fractionnement nécessite des dépenses supplémentaires dans la conduite de la culture qu'il faut compenser normalement avec le surplus de récolte qu'induit ce fractionnement.

Technique no. 1, Ministère Agriculture et Elevage, Ouagadougou, Burkina Fa Chougar S., 2011. Bioécologie de la mineuse de la tomate Tuta absoluta (Lepidoptera: Gelechiidae) sur trois variétés de tomate sous serre (Zahra, Dawson et Tavira) dans wilaya de Tizi- Ouzou. Mém. De Magister, Univ. Mouloud Mammeri de Tizi- Ouzou, (2011)106p.

Djidji A.H., Zohouri G.P., Fondio L., NZI J. C. et Kouamen C., (2010). Effet de l'abri sur le comportement de la tomate (Solanum lycopersicum L.) en saison pluvieuse dans le Sud de la Côte- 
d'Ivoire. Journal of Applied Biosciences, 25:1557 - 1564, CNRA Côte D'Ivoire, ISSN 1997-5902, 8p.

FAO, IFA et IMPHOS, 2003. Les engrais minéraux et leurs applications. Précis à l'usage des agents de vulgarisation agricole. Quatrième édition, version révisée, publiée par la FAO, l'IFA et l'IMPHOS, ISBN 92-5-104414-7, 77p.

Galla Bi T.J., Camara M., Yao Kouamé A. et Keli Z.J., 2011. Rentabilité des engrais minéraux en riziculture de plateau : cas de la zone de Gagnoa dans le centre ouest de la Cote d'Ivoire. Journal of applied bioscience, 46 : 3153-3162.

Ganry F., 1990. Application de la méthode isotopique à l'étude des bilans azotés en zone tropicale sèche. Thèse: Sciences Naturelles; univ. de Nancy 1: 354p.

Gigou J. et Chabalier C.F., 1987. L'utilisation de l'engrais azoté par les cultures annuelles en Côte d'Ivoire. Agronomie Tropical, 42 (3)171-175.

Guinko S., 1984. Végétation de la haute volta. Thèse de Doctorat d'Etat Sciences Naturelles. Univ. de Bordeaux III, (1984) 318p.

INERA/CREAF., 2010. Evaluation variétale de tomates industrielles dans deux sites (Loumbila et Kamboinsé) pour la culture au Burkina. Rapport, INERA/CREAF de Kamboinsé et STFLL, (2010) 43p.

Institut Agronomique et Vétérinaire Hassan II (IAV), 1999. Plantes, Végétaux, Advances, 17 (2-3)183-203.

Jacob J. P. et. Janssen J. L.M., 1979. La tomate in : cultures maraîchères spéciales: les solanacées fruits. Cours polycopies. INA. El-Harrach., (1979) 01-16.

Kabrahy R., Yao N., Dea G. B. et Couloudi Y., 1996. Effet de l'apport d'engrais et de matière organique sur le rendement en grains chez le maïs. Cabiers Agriculture, 5: 189 - 93.

Kaloga B., 1969. Etude de la pédogenèse sur les glacis soudaniens de Haute-Volta, Bulletin de Liaison - ASEQUA, (22) 20 23.
Kolev N., 1976. Les cultures maraîchères en Algérie. Tome I: légumes fruits. Ed. Ministère de l'Agriculture et de la Réforme Agraire (MARA), 1: 52p.

Lafond J., 2004. Fractionnement de la fertilisation azotée minérale et organique: Effet sur la productivité du canola de printemps et sur les nitrates du sol. Can. J. Soil Sci, 84:491-501.

Laumonnier R., 1979. Cultures légumières et maraîchères. Tome III. Ed. J. B. Baillière Paris, 274p.

MAHRH., 2007. Analyse de la filière maraîchère au Burkina Faso. Edit DGPSA, Burkina Faso, 127p.

Marques A. et Moreau M., 2007. La tomate sous abri. BTSA PC 2005- 2007, 3p.

MASA., 2012. Rapport d'analyse du maraîchage: Campagne 2011/2012. Edit. DGESS, $42 \mathrm{p}$.

Nyembo K.L., Useni S.Y., Chukiyabo K.M., Tshomba K.J., Ntumba M.E., Muyambo K.P., Kapalanga M.M., Mpundu M.D., Bugeme N.F. et Baboy L.L., 2013. Rentabilité économique du fractionnement des engrais azotés en culture de maïs (Zea mays L.): cas de la ville de Lubumbashi, sud-est de la RD Congo. Journal of Applied Biosciences, 65 (2013) 4945 - 4956.

Pieri C., 1982. Estimation du bilan des pertes moyennes en eaux et en éléments minéraux dans une succession culturale mil - arachide. Rapport de mission, 26p.

PIP et COLEACP, 2011. Itinéraire technique tomate cerise (Lycopersicon esculentum WWW.coleacp.or/pip.

Polese K.M., 2007. La culture de tomate. Ed. Artémis, 95p.

Sedego P.M., Bado B.Y., Cescas M. P., Lompo F. et Bationo A., 1997. Effet à long terme des fumures sur les sols et les rendements du maïs au Burkina Faso. Cabier d'Agriculture, $6: 571-575$.

Shankara N., Van lidt De jeud J., De Goffau M., Hilmi M., Van Dam B. et Florijin A., 2005. La culture de la tomate : 
production, transformation et commercialisation. 5eme (ed). foundation agromisa et CTA, Wageningen. Pays- Bas, 105p.

Tirilly Y. et Bourgeois C.M., 1999. Technologie des légumes, In Philouze J, La tomate et son amélioration génétique. Ed., Tec \& Doc., (1999) 112-130.

Ziang H., 1984. Les aléas climatiques liés à la production quantitative et qualitative de la tomate. In, Agrométéorologie et productions légumières, séminaire tenu a Avignon du 19 février au 1è mars 1984, les colloques de L'INRA, Paris, 33: $77-79$. 